\title{
Sicurezza e tollerabilità della somministrazione di iloprost senza pompa peristaltica
}

\section{Infusion of iloprost without a peristaltic pump: Safety and tolerability}

\section{Paola Faggioli, Leopoldo Giani, Antonino Mazzone*}

\author{
UO Medicina Interna, AO Ospedale Civile di Legnano - Presidio di Legnano, Milano
}

Ricevuto il 9 aprile 2010; accettato il 16 aprile 2010

disponibile online il 6 maggio 2010

\section{KEYWORDS \\ Iloprost; \\ Safety; \\ Peristaltic pump; \\ Systemic sclerosis; \\ Peripheral arterial \\ disease; \\ Infusion-related \\ adverse events.}

\begin{abstract}
Summary
Introduction: Iloprost is a potent prostacyclin (PGI2) analogue that is effective in the treatment of peripheral arterial disease, vasculitis, pulmonary hypertension, and secondary Raynaud's phenomenon. Intravenous infusions are generally administered with the aid of a peristaltic pump to reduce the risk of adverse reactions caused by unintentional increases in the infusion rate. This increases the cost of care in terms of equipment and personnel and may limit the use of this drug. Materials and methods: We retrospectively analyzed 18,432 iloprost infusions administered between 1999 and 2009 to 272 patients with systemic sclerosis $(n=253)$ and 19 with peripheral arterial disease $(n=19)$. All infusions were administered in the day hospital over $6 \mathrm{~h}$ with a normal IV set-up with a roller flow regulator. Flow rates were set to deliver iloprost at $1-2 \mathrm{ng} / \mathrm{kg} / \mathrm{min}$. Rates were verified by direct drop counts during the first 15-20 minutes of the infusion and at each subsequent check.

Results: There were no adverse events that were fatal, life-threatening, or associated with prolongation of hospitalization and very few events requiring intensive care or continuous monitoring. The latter included 4 cases of tachycardia/arrhythmia (extrasystoles in most cases), 3 cases of hypotension (systolic pressure $<80 \mathrm{mmHg}$ ), and 2 cases of hypertension (BP $>$ $170 / 100 \mathrm{mmHg}$ ). All other adverse reactions were mild, reversible, and similar to those seen with iloprost infusion with peristaltic pump. Only one patient had to be switched to another prostanoid (due to intolerance).

Discussion: Iloprost infusion administered with a normal IV flow regulator appears to be as safe, well tolerated, and effective as traditional infusion with a peristaltic pump.

(c) 2010 Elsevier Srl. All rights reserved.
\end{abstract}

\footnotetext{
* Corrispondenza: UO Medicina Interna, (DH) Presidio di Legnano, AO Ospedale Civile di Legnano, via Candiani 2 - 20025 Legnano, MI. E-mail: medicina2legnano@ao-legnano.it (A. Mazzone).
} 


\section{Introduzione}

L'iloprost è un analogo sintetico stabile della prostaciclina naturale (PGI2), sintetizzato nel 1978, che a differenza della prostaciclina naturale, che presenta una emivita molto ridotta (ca 2-3 min), ha una emivita più lunga che consente il suo utilizzo terapeutico.

\section{Cenni sui prostanoidi}

La PGI2 viene sintetizzata principalmente a livello delle cellule endoteliali a partire da una sintesi enzimatica da parte della prostaciclina sintetasi (PGIS) [1] e delle cicloossigenasi (COX1 principalmente COX2) che sono soprattutto localizzate a livello del reticolo endoplasmatico e delle membrane nucleari delle cellule endoteliali, e delle cellule muscolari lisce dei vasi sanguigni in varia misura. La loro presenza anche a livello delle membrane nucleari di tali cellule ne suggerisce anche un ruolo non soltanto esocrino, ma anche paracrino e autocrino che potrebbe spiegare $i$ diversi effetti metabolici e regolatori della prostaciclina [2-4].

Dapprima i recettori per la PGI2 sono stati principalmente localizzati a livello delle superfici cellulari endoteliali (IP) di cui sono stati descritti tre sottotipi con affinità differente e diversa espressione genica, in grado quindi di determinare la risposta biologica sia alla PGI2 naturale che all'iloprost [5].

Un secondo gruppo di recettori è stato identificato a livello intracellulare PPARs (peroxisome, proliferator-activated receptors), presenti ubiquitariamente a livello cellulare e non solo a livello endoteliale, che spiegherebbero le numerose attività metaboliche della prostaciclina, mediate dall'attivazione o dalla repressione genica. L'iloprost interagisce con entrambi i recettori e proprio grazie a questa interazione si possono spiegare le sue azioni di regolazione genica e le sue proprietà terapeutiche [6-9].

$\downarrow$ CTGF (riduzione della fibrogenesi).

$\downarrow$ MCP-1 (riduzione della chemiotassi dei monociti).

$\downarrow$ PAl-1 (attività profibrinolitica, che viene mantenuta

long-term).

$\uparrow$ VEFG (promozione della neoangiogenesi).

$\uparrow$ TGF-Beta (effetto antinfiammatorio).

$\uparrow$ COX2 (regolazione della ciclo ossigenasi).

L'iloprost trova quindi la sua indicazione nel trattamento delle principali patologie vascolari di tipo ischemico, quali l'arteriopatia obliterante periferica [10], m. di Buerger $[11,12]$ il fenomeno di Raynaud secondario a sclerosi sistemica [13], l'ipertensione polmonare [14]. Nuove prospettive terapeutiche si stanno aprendo grazie agli studi clinici tuttora in corso, come nelle ulcere vasculitiche, nelle sindromi da iperviscosità [15], nell'embolia periferica da cristalli di colesterolo (blu toes syndrome) [16], nel trattamento delle ulcere venose [17], nell'osteonecrosi asettica [18], nel pioderma gangrenoso [19] e come coadiuvante nella terapia dell'insufficienza renale acuta e nelle crisi renale sclerodermica [20], nel post trapianto [21] e negli stadi precoci delle arteriopatie periferiche e diabetiche [22-24].

Eccettuata la via inalatoria, riservata al trattamento dell'ipertensione polmonare, ed eccezionalmente la via intra-arteriosa, a causa della sua breve emivita plasmatica (emivita di distribuzione tissutale 4 minuti), l'iloprost viene somministrato con un'infusione endovenosa della durata di 6 ore, raggiungendo lo steady state in 20 minuti, mentre l'emivita di eliminazione è di circa 20-30 minuti. L'escrezione è per 1 ' $80 \%$ a livello urinario e per il $20 \%$ a livello epatico, sotto forma di vari metaboliti (di cui il principale è il tetranoriloprost farmacologicamente inattivo), dotati di una emivita di 2-5 ore a livello plasmatico e 2-18 ore a livello urinario.

Secondo lo schema posologico approvato dalle autorità regolatorie, iloprost deve essere infuso a una velocità di 0,5-2 $\mathrm{ng} / \mathrm{kg} / \mathrm{min}$ per almeno 6 ore continuative, partendo da $0,5 \mathrm{ng} / \mathrm{kg} / \mathrm{min}$ e salendo gradatamente ogni 30 minuti fino a $2 \mathrm{ng} / \mathrm{kg} / \mathrm{min}$ o al massimo dosaggio tollerato. Gli schemi terapeutici sia per durata dei cicli infusionali sia per periodicità variano a secondo della patologia trattata.

Comunemente viene consigliato l'uso di una pompa peristaltica per infusione che, garantendo una velocità costante e controllabile, ridurrebbe il rischio di reazioni avverse al farmaco legate a possibili anomali picchi infusionali. Tale metodica, tuttavia, richiede necessariamente un maggiore carico assistenziale ed economico del reparto, limitandone di conseguenza la possibilità di un suo impiego.

L'infusione in 6 ore della dose terapeutica giornaliera di iloprost, mediante un comune deflussore con regolatore di flusso, potrebbe ampliarne le possibilità di impiego, in quanto richiede minori costi gestionali e assistenziali, permettendone la somministrazione in regime di Day Hospital (DH) o ambulatoriale.

Iniziali studi condotti sull'infusione di iloprost mediante deflussore con regolatore di flusso in casistiche limitate di pazienti hanno confermato una efficacia terapeutica e un profilo di sicurezza sovrapponibile alla infusione mediante pompa peristaltica $[25,26]$.

Scopo del nostro studio è stato quindi valutare in una vasta casistica di pazienti, raccolta nel corso di dieci anni di impiego di questa metodica, il profilo di sicurezza della infusione in 6 ore della dose terapeutica giornaliera di iloprost mediante deflussore con regolatore di flusso.

\section{Materiali e metodi}

Sono stati ammessi alla terapia pazienti affetti da fenomeno di Raynaud secondario a sclerosi sistemica progressiva (SSP), sia nella variante diffusa sia limitata, con un numero di attacchi medio giornaliero di tre nei mesi invernali o con lesioni trofiche digitali, e pazienti affetti da 'arteriopatia obliterante cronica periferica' (AOCP) in stadio III-IV di Fontaine.

Non sono stati ammessi alla terapia pazienti che presentavano le seguenti caratteristiche: rifiuto alla terapia, stato di gravidanza o allattamento, fumo attivo, cardiopatia ischemica con infarto miocardico antecedente a sei mesi, scompenso cardiocircolatorio acuto in classe III-IV NYHA, aritmie non controllate; mentre non sono stati considerati come criteri di esclusione la presenza di ipertensione arteriosa purché controllata dalla terapia, concomitante terapia anticoagulante orale o antiaggregante, insufficienza renale o insufficienza epatica moderata. La concomitante presenza di ipertensione polmonare che si è osservata in 10 pazienti affetti da SSP ha posto indicazione a trattamento in associazione con inibitori recettoriali dell'endotelina (ERA) e con 
sildenafil senza che si siano riscontrate reazioni avverse significative.

Prima dell'infusione tutti i pazienti sono stati accuratamente valutati al fine di escludere la presenza dei fattori di esclusione e, in particolare, sono stati tutti sottoposti a valutazione cardiologica ed esame elettrocardiografico. II regime terapeutico prevedeva che i pazienti continuassero la terapia abituale, adottando la precauzione di rimandare l'assunzione di calcio antagonisti al termine dell'infusione per evitare sinergie con l'iloprost nel controllo dei valori pressori.

Tutti i pazienti sono stati ricoverati in regime di $\mathrm{DH}$, dove sono stati monitorizzati a cadenza oraria i parametri vita principali (pressione arteriosa, frequenza cardiaca) ed è stata rilevata l'eventuale insorgenza di reazioni avverse.

L'iloprost (Endoprost ${ }^{\circledR}$, Italfarmaco SpA, Italia), disponibile in fiale da $0,5 \mathrm{ml}$ contenenti $0,05 \mathrm{mg}$ di principio attivo, veniva diluito in $250 \mathrm{ml}$ di soluzione fisiologica e veniva somministrato a un dosaggio iniziale di $0,5 \mathrm{ng} / \mathrm{kg} / \mathrm{min}$ aumentando ogni 30 minuti la velocità infusionale fino a ottenere o la massima velocità tollerata dal paziente, o la velocità massima di infusione di $2 \mathrm{ng} / \mathrm{kg} / \mathrm{min}$.

Abbiamo impiegato schemi differenti a secondo delle differenti patologie trattate. Nella SSP dopo un primo ciclo di 5 giorni in cui veniva individuato il massimo dosaggio tollerato di iloprost, la somministrazione veniva ripetuta mensilmente, a eccezione dei mesi estivi (luglio-agosto), nei pazienti clinicamente stabili.

Nella AOCP i cicli avevano una durata di 15-20 giorni e venivano ripetuti mediamente tre volte all'anno.

In entrambe le patologie la durata dell'infusione non è mai stata inferiore alle 6 ore.

Per la somministrazione è stato usato un sistema infusionale in polivinilcloruro comprendente un perforatore per flaconi con cono di deflusso collegato a un tubo dotato di un sistema di regolatore di flusso "roller" che veniva raccordato con un regolatore di flusso per soluzioni iv (Euroset system, Eurospital, Trieste, Italia). Tale regolatore era dotato di una scala di taratura in $\mathrm{ml} /$ ora (su doppia scala a secondo della viscosità della soluzione) e veniva connesso al sistema di venipuntura (caterere venoso di $22 \mathrm{~F}$, NEO VENOTROPIC 2 in FEP, G22 $42 \mathrm{ml} / \mathrm{min}$ - Artsana SPA ComoGrandate). Il paziente era adeguatamente istruito sulla non manomissione autonoma del deflussore, che veniva impostato sulla velocità di infusione desiderata $(1-2 \mathrm{ng} / \mathrm{kg} / \mathrm{min}$ di iloprost). Poiché la velocità di infusione poteva essere influenzata dalla forza di gravità, dalla postura del braccio del paziente e dalla portata venosa, nei primi 15-20 minuti dell'infusione e nei controlli infermieristici successivi veniva effettuato un controllo della velocità indicata dal regolatore di flusso, anche mediante il conteggio diretto delle gocce.

Sono stati valutati in corso di infusione l'insorgenza di reazioni avverse, e i provvedimenti terapeutici adottati. Prima e durante l'infusione è stato eseguito il monitoraggio dei valori pressori. I valori pressori erano anche valutati alla sua sospensione per valutare un possibile effetto rebound, verosimilmente imputabile a una iperattivazione colinergica.

Tutti i pazienti avevano firmato un adeguato consenso informato illustrante $i$ benefici e le possibili reazioni avverse collaterali legate alla terapia con iloprost. Una dichiarazione liberatoria ai fini dell'uso dei dati personali in forma anonima secondo la nuova normativa della privacy è stata successi- vamente somministrata e firmata. II Comitato Etico locale è stato informato della possibilità di uno studio osservazionale spontaneo non profit con i dati ottenuti e non ha espresso parere contrario nei tempi prestabiliti, secondo la normativa del silenzio-assenso.

\section{Risultati}

Nel periodo di tempo compreso dal 1999 al 2009 presso I'istituto di Medicina Interna del Policlinico S. Matteo di Pavia e I'UO di Medicina Interna dell'Ospedale Civile di Legnano sono osservati consecutivamente 272 pazienti, tutti sottoposti a 6 ore di infusione con iloprost mediante microgocciolatore per un totale di 18.432 infusioni complessive.

Le caratteristiche dei pazienti sono esposte nella tabella 1.

Tutti i 272 pazienti sono stati sottoposti almeno a una infusione di iloprost. Ogni paziente affetto da SSP è stato sottoposto a 10-14 infusioni/anno (valore mediano 12), mentre nelle AOCP sono state praticate da 30 a 45 infusioni/anno (valore mediano 41).

Non è stata segnalata dai pazienti o osservata dal personale medico o infermieristico nessuna reazione avversa classificabile come seria (cioè che induce a morte, pericolo di vita, causa o prolunga l'ospedalizzazione ecc.) e inattesa.

Tutte le tipologie di reazioni avverse osservate sono descritte nel RCP del prodotto.

La percentuale di incidenza di reazioni infusionali che abbiamo classificato come gravi e cioè tali da richiedere un supporto terapeutico intensivo o un monitoraggio continuativo dei parametri vitali appare trascurabile: esse sono consistite in casi di tachicardia o aritmia (più frequentemente extrasistolia), ipotensione (PA sistolica $<80 \mathrm{mmHg}$ ), 0 ipertensione (PA $>170 / 100 \mathrm{mmHg}$ ), come indicato in tabella 2.

Sono state quindi analizzate le reazioni avverse non gravi (reazioni che non hanno richiesto un trattamento medico intensivo o un monitoraggio continuativo dei parametri vitali

Tabella 1 Caratteristiche dei pazienti.

\begin{tabular}{ll}
\hline Pazienti (N.) & 272 \\
Sesso & $225 / 47$ \\
Età, anni (mediana) & $61,2 \pm 11,4(54,9)$ \\
Diagnosi & \\
$\quad$ SSP & $253(93,01 \%)$ \\
AOCP & $19(6,9 \%)$ \\
\hline
\end{tabular}

$\mathrm{AOCP}=$ arteriopatia obliterante cronica periferica; SSP = sclerosi sistemica.

Tabella 2 Reazioni avverse gravi in corso di infusione.

\begin{tabular}{lll}
\hline $\begin{array}{l}\text { Tipo di reazione avversa in corso } \\
\text { di infusione }\end{array}$ & $\%$ & N. pazienti \\
\hline Tachicardia (> 150 bpm)/aritmia & 1,5 & 4 \\
Ipotensione (PA sist. $>80 \mathrm{mmHg}$ ) & 0,8 & 3 \\
lpertensione sisto-diastolica & 0,7 & 2 \\
(PA $>170 / 100 \mathrm{mmHg})$ & & \\
\hline
\end{tabular}


Tabella 3 Reazioni avverse non gravi in corso di infusione.

\begin{tabular}{ll}
\hline Tipo di reazione avversa & $\%$ \\
\hline Cefalea & 70,1 \\
Trisma flushing & 56,3 \\
Flushing & 52,2 \\
Nausea (tollerabile) & 34,7 \\
Vomito & 14,8 \\
Fenomeno di Raynaud & 9,7 \\
Diarrea & 4,9 \\
Iperemia lungo la vena incanulata & 1,4 \\
\hline
\end{tabular}

e che si sono risolte nel giro di 5 minuti dalla sospensione del farmaco e non ne hanno pregiudicato le successive infusioni) insorte durante la somministrazione del farmaco, calcolando la relativa percentuale di incidenza (tabella 3 ).

Tutte le reazioni si sono risolte dopo il termine dell'infusione, alcune di esse hanno richiesto un aggiustamento posologico o il rallentamento della velocità infusionale. Nessuna di esse ha portato alla sospensione della terapia.

Sono state valutate anche le reazioni post-infusionali, considerandone l'incidenza e la durata (tabella 4). I provvedimenti adottati al fine di controllare la sintomatologia collegata alle reazioni avverse sono stati finalizzati al trattamento dei sintomi gastroenterici, della cefalea e dei disturbi ipertensivi come evidenziato nella tabella 5. Tali trattamenti, ritenuti efficaci nel controllo dei sintomi, sono stati adottati con successo anche come premedicazione del paziente nei cicli successivi al primo, con il risultato di aumentare la compliance alla terapia.

La sospensione del trattamento è avvenuta in 18 pazienti (tabella 6). In un solo caso, essa è stata causata da intolleranza al farmaco, che è stato quindi sostituito da un altro prostanoide.

In due pazienti affetti da sclerosi sistemica e ipertensione polmonare secondaria in stadio avanzato e in terapia di associazione con ETRA e inibitori della fosfodiesterasi, si è sospeso iloprost, passando al trattamento con epoprostenolo in infusione continua.

In 3 pazienti ultraottantenni, la graduale insorgenza di una cardiomiopatia senile con labile compenso è stata ritenuta una controindicazione alla terapia con iloprost, e ciò ha motivato il passaggio a trattamento con cilostazolo.

\section{Discussione}

Le nostre osservazioni, condotte su una casistica di pazienti molto più ampia rispetto a quanto riportato fin ora in letteratura, suggeriscono che la somministrazione di iloprost mediante deflussore con regolatore di flusso è sicura e offre gli stessi vantaggi della somministrazione mediante pompa

Tabella 4 Reazioni post infusionali.

\begin{tabular}{|c|c|c|c|}
\hline $\begin{array}{l}\text { Reazione post- } \\
\text { infusionale }\end{array}$ & $\begin{array}{l}\text { Frequenza } \\
(\% \pm D S)\end{array}$ & $\begin{array}{l}\text { Durata } \\
(\min \pm D S)\end{array}$ & Trattamento \\
\hline Astenia & $55,3 \pm 3,2$ & $120 \pm 60$ & Nessuno \\
\hline Vertigine/Confusione & $35,5 \pm 2,2$ & $95 \pm 60$ & Nessuno \\
\hline Rebound pressorio & $6,4 \pm 1,0$ & $45 \pm 15$ & Nessuno \\
\hline
\end{tabular}

Tabella 5 Provvedimenti al fine di ridurre l'incidenza e la gravità delle reazioni avverse.

\begin{tabular}{ll}
\hline Reazione avversa & Provvedimento (\%) \\
\hline Nausea - Vomito & Metoclopramide ev (90\%) \\
& Ondansetron ev (8\%) \\
& Metoclopramide im (2\%) \\
\hline Cefalea & Paracetamolo os (36,4\%) \\
& Ketorolac os (22,3\%) \\
& Ketorolac ev (20,7\%) \\
& Paracetamolo ev (20,6\%) \\
\hline Ipotensione & Calcio gluconato 1 fl \\
& ev + sospensione infusione (90\%) \\
& Sospensione infusione (10\%) \\
\hline Ipertensione & Sospensione infusione (70\%) \\
& Sospensione infusione + clonidina \\
& im o iv (15\%) \\
\hline Altro & Riduzione della dose infusa (20,6\%) \\
& Riduzione della velocità di \\
& infusione (14,7\%) \\
& Passaggio a infusione con pompa \\
volumetrica (2\%)
\end{tabular}

peristaltica, con una incidenza di effetti collaterali del tutto sovrapponibili a quanto riportato in letteratura $[25,26]$. Nella quasi totalità dei casi osservati è stato raggiunto il dosaggio terapeutico di iloprost, assestato mediamente attorno a 1,5$2 \mathrm{ng} / \mathrm{kg} / \mathrm{min}$.

Generalmente l'insorgenza di reazioni avverse, peraltro di gravità tale da non pregiudicare le condizioni dei pazienti, ha avuto una incidenza maggiore $(75 \%)$ al primo ciclo infusionale. Tali reazioni si sono costantemente risolte al termine dell'infusione.

Nelle infusioni successive, le reazioni avverse tendevano a ripresentarsi con le stesse modalità in ciascun paziente, anche se in forma più lieve e con minore durata. Secondo la nostra esperienza tale comportamento non è da collegarsi a una tolleranza indotta al farmaco, quanto alla terapia farmacologica di supporto adottata al fine di limitare le reazioni avverse, alla personalizzazione del dosaggio e della velocità infusionale. Questo ha sicuramente aumentato anche la compliance dei pazienti alla terapia e ha portato a un incremento nel tempo del numero delle infusioni per paziente.

Curiosamente la maggior parte delle reazioni infusionali si sono manifestate nei pazienti affetti da SSP ( $96 \%$ vs $4 \%$ ); una possibile spiegazione potrebbe essere ricondotta alla cardiopatia sclerodermica seppure silente, che espone tali pazienti a un maggior rischio di insorgenza di aritmie e di puntate ipertensive. Del resto non è stata documentata nei pazienti sclerodermici una alterazione della clearance dell'iloprost, a differenza di quanto riportato in sporadiche osservazioni condotte su pazienti arteriopatici [27].

Appaiono relativamente frequenti l'insorgenza di astenia, imputabile verosimilmente all'effetto vasodilatatore dell' $i$ loprost e alla degenza ospedaliera, come del resto sembrano correlabili a vasodilatazione e ad attivazione vagale sia la sindrome vertiginosa sia il senso di confusione/deficit di attenzione lamentati. 
Tabella 6 Sospensione del trattamento (tot. 18 pz.).

\begin{tabular}{llll}
\hline Motivazione & N. pz $(\%)$ & Temporanea/definitiva & Note \\
\hline Intolleranza a iloprost & $1(0,5)$ & Definitiva & Passaggio ad alprostadil \\
\hline Decesso & $4(2,1)$ & Definitiva & Peggioramento della sclerodermia \\
\hline Neoplasia & $6(3,1)$ & $\begin{array}{l}\text { Definitiva }(3) \\
\text { Temporanea }(3)\end{array}$ & $\begin{array}{l}\text { Mantenimento della terapia } \\
\text { per peggioramento del FR }\end{array}$ \\
\hline PAH IV stadio NYHA & $2(1,0)$ & Definitiva & Passaggio a epoprostenolo \\
\hline Età avanzata ( $>80$ aa.) comorbidità & $3(1,6)$ & Definitiva & Passaggio a cilostazolo \\
\hline Rifiuto del paziente per ragioni logistiche & $2(1,0)$ & Definitiva & Passaggio a pentosifillina
\end{tabular}

Precauzionalmente, pur non essendoci dati pre-clinici sulla oncogenicità o sull'effetto mutageno di iloprost si è preferito sospendere la terapia in alcuni pazienti che hanno sviluppato neoplasie (peraltro non correlabili al farmaco) per lo meno durante i cicli chemioterapici in considerazione del suo effetto pro-angiogenetico.

La valutazione dell'efficacia terapeutica di iloprost non era tra gli end points del nostro studio. Si può comunque affermare che si è ottenuto un miglioramento nel $78,9 \%$ dei pazienti sclerodermici in termini di riduzione del numero di attacchi e della durata del fenomeno di Raynaud [28]. Tale miglioramento ha mostrato una tendenza all'aumento della risposta nei cicli successivi [29].

Un miglioramento dell'intervallo libero di marcia è stato osservato nel $77 \%$ dei pazienti affetti da AOCP in stadio III e nel $49 \%$ dei pazienti in stadio IV, mentre la diminuzione del dolore a riposo è stata rispettivamente dell' $83 \%$ e nel $49 \%$ dei pazienti in stadio IV, mentre la diminuzione del dolore a riposo è stata rispettivamente dell' $83 \%$ e del $58 \%$ nell'AOCP stadio III e IV (tabella 7).

La quantità di soluzione rimasta al termine delle infusioni (prendendo come standard una durata complessiva di 6 ore), è stata valutata in circa 3.600 infusioni (20\%). Il residuo non infuso era di $5 \pm 2 \mathrm{ml}$, quantità da considerare irrilevante ai fini dosaggio della dose terapeutica quotidiana infusa.

I nostri risultati sembrano quindi riconfermare la sicurezza, la buona tollerabilità e l'ottima compliance della somministrazione di iloprost, anche quando somministrato

Tabella 7 Efficacia terapeutica.

\begin{tabular}{ll}
\hline Parametri di efficacia terapeutica & $\begin{array}{l}\text { Percentuale di } \\
\text { pazienti che hanno } \\
\text { riscontrato un } \\
\text { miglioramento }\end{array}$ \\
\hline $\begin{array}{l}\text { Riduzione del numero di attacchi } \\
\text { e della durata del fenomeno } \\
\text { di Raynaud }\end{array}$ & $78,9 \%$ \\
\hline $\begin{array}{l}\text { Aumento dell'Intervallo Libero di Marcia: } \\
\text { - in paz. con AOCP in stadio III }\end{array}$ & $77 \%$ \\
- in paz. con AOCP in stadio IV & $49 \%$ \\
\hline Riduzione del Dolore a riposo: & \\
- in paz. con AOCP in stadio III & $83 \%$ \\
- in paz. con AOCP in stadio IV & $58 \%$ \\
\hline
\end{tabular}

mediante sistema infusionale con deflussore e regolatore di flusso, a parità di efficacia clinica.

Tale modalità, oltre a essere economicamente più vantaggiosa, offre maggiori possibilità di impiego dell'iloprost anche in regime di $\mathrm{DH}$ o ambulatoriale permettendo un allargamento delle possibilità di utilizzo del farmaco e garantendo quindi una ulteriore possibilità terapeutica a un numero crescente di pazienti.

\section{Conflitto di interesse}

Gli autori dichiarano di essere esenti da conflitto di interessi.

\section{Bibliografia}

[1] Wada M, Yokoyama C, Hatae T, Shimonishi M, Nakamura M, Imal $Y$, et al. Purification and characterization of recombinat human prostacyclin syntase. J Biochem 2004;135(4):455-63.

[2] Lim H, Dey SK. Minireview: a novel pathway of prostacyclin signaling-hanging out with nuclear receptors. Endocrinology 2002;143(9):3207-10.

[3] Liou JY, Shyue SK, Tsai MJ, Chung CL, Chu KY, Wu KK. Colocation of prostacyclin synthase with prostaglandin $\mathrm{H}$-synthase 1 but not phorbol ester-induced PGHS-2 in cultured endothelial cells. J Biol Chem 2000;19:15314-20.

[4] Narumiya P, Sugimoto Y, Ushikubi F. Prostanoid receptors: structures, properties and functions. Physiol Rev 1999;79:1193-226.

[5] Funk CD. Prostaglandins and leukotriens: advances in eicosanoid biology. Science 2001;294:18721-5.

[6] Katsuyama M, Surgimoto Y, Namba T, Irie A, Negishi M, Narumiya $\mathrm{S}$, et al. Clonig and expression of c-DNA for the human prostacyclin receptor. FEBS lett 1994;344:74-8.

[7] Pola R, Gaetani E, Flex A, et al. Comparative anlysis of the in vivo angiogenic properties of stable prostacyclin analogs: a possible role for peroxisome prolipherator activated receptors. J Mol Cell Cardiol 2004;36:363-70.

[8] Toshihisa H, Masayuki W, Yokoyama C, Shimonishi M, Tanabe T. Prostacyclin dependent apoptosis mediated by PPAR. J Biol Chem 2001;276:46260-7.

[9] Banyai S, Jenelten R, Wagner S, Allmann J, Banyai $M$, Koppensteiner R. Outpatient treatment of severe peripheral ischemia with intravenous intermittent low dose iloprost. An open pilot study. Int Angiol 2002;21:36-43.

[10] Cipollone F, Fazia M, Mincione G, lezzi A, Pini B, Cuccurullo F, et al. Increased expression of Transforming Growth Factorbeta1 as stabilizing factor in human atherosclerotic plaques. Stroke 2004;67:757-65. 
[11] Norgren L, Hiatt WR, Dormandy JA, Nehler MR, Harris KA, Fowkes FG, et al., TASC II Working Group. Int Angiol 2007;26(2):81-157.

[12] Hirsch AT, Haskal ZJ, Hertzer NR, Bakal CW, Creager MA, Halperin JL, et al. ACC/AHA 2005 Practice Guidelkines for the Management of Patients with Periferal Arterial Disease (lower extremity renal mesenteric and abdominal aortic). Circulation 2006;113(11):e463-564.

[13] Scorza R, Caronni M, Mascagni B, Berruti V, Bazzi S, Micallet E, Arpaia G, et al. Effect of long-term cycling iloprost therapy in Systemic Sclerosis with raynaud's phenomenon. A randomized, controlled study. Clin Exp Rheumatol 2001;19:503-8.

[14] Olschewski H, Hoeper MM, Behr J, Ewert R, Meyer A, Borst MM, Winkler J, et al. Long-term therapy with inhaled iloprost in patients with pulmonary hypertension. Respir Med 2010 Feb 10 [Epub ahead of print].

[15] Zulian F, Costantini C, Montesco MC, Schiavon F, Zacchello F. Successful treatment of gangrene in sistemi necrotizing vasculitis with iloprost. Br J Rheumatol 1998;37:228-30.

[16] Meyrier A. Cholesterol crystal embolism: diagnosis and treatment. Kidney Int 2006;69(8):1308-12.

[17] Ferrara F, Meli F, Raimondi F, Amato C, Bonura F, Mulè G, Novo G, Novo SS. The treatment of venous leg ulcers. A new therapeutic use of iloprost. Ann Surg 2007;246(5):860-5.

[18] Jager M, Tillmann FP, Thomhill TS, Mahmoudi M, Blondin D, Hetzel GR, et al. Rationale for prostaglandin 12 in bone marrow oedema - from theory to application. Arthritis Res Ther 2008;10(5):R120.

[19] Mazzone A, Cusa C, Mazzucchelli I, Rosso R, Vezzoli M. Pyoderma gangrenosus complicating ulcerative colitis: an alternative treatment with iloprost. Current Therapeutic Research 1999;60:357-62.

[20] Scorza R, Rivolta R, Mascagni B, Berruti V, Bazzi S, Castagnone $D$, Quarto di Palo F. Effect of iloprost infusion on the resistence index of renal vessels of patients with systemic sclerosis. J Rheumatol 1997;24:1944-8.
[21] Knight KR, Lepore DA, O’Brien BM. Interrelationschips between prostanoid and skin flap survival: a review. Prostaglandins Leukot Essent Fatty Acids 1991;44(4):195-200.

[22] Duthois S, Cailleux N, Benosman B, Levesque H. tholerance of iloprost and results of treatment of chronic severe lower limb ischaemia in diabetic patients. A retrospective study of 64 consecutive cases. Diabetes Metab 2003;29(1):36-43.

[23] Meini S, De Franco V, Auteri A, Setacci C, Di Renzo M, Pieragalli D. Short term effects of one-week treatment with intravenous iloprost in critical limb ischemia patients (lerich Fontaine stage III and IV). Int Angiol 2005;24(1):64-9.

[24] Veroux P, Veroux M, Macarrone M, Bonanno MG, Tumminelli MG. Efficacy of a novel method of intravenous infusion of the prostaglandin analogue iloprost for the treatment of lower limb ischemia: an open label, non randomized study in two cohorts. Curr Ther Res Clin Exp 2004;65:255-65.

[25] Mazzone A, Vezzoli M, Ottini E, Montagna M, Mazzucchelli I, Dal Canton A. A new method of iloprost administration without a peristaltic pump. Curr Ther Res 2000;61:452-9.

[26] Arreghini M, Prudente P, Maglione W, Arnoldi S, Tosi S, Marchesoni A. Tollerabilità, sicurezza ed efficacia del trattamento infusivo con iloprost senza pompa peristaltica in pazienti affetti da sclerosi sistemica. Reumatismo 2001; 53(2):140-4.

[27] Hildebrand $M$, Krause W, Oberender HA, Zurdel-Dillinger S, Junger $\mathrm{M}$, Bodenburg $\mathrm{H}$. Pharmacokinetic of iloprost in patients with severe peripheral arterial occlusive disease. Eicosanoids 1990;3(3):145-8.

[28] Milio G, Corrado E, Genova C, Amato C, Raimondi F, Almasio PL, et al. Iloprost treatment in patients with Raynaud's phenomenon secondary to systemic sclerosis and the quality of life. A new therapeutic protocol. Rheumatology 2006;45(8):9991004.

[29] Duthois S, Cailleux N, Lavesque H. Tolerance and therapeutic results of iloprost in obliterative arteriopathy in lower limbs at the severe chronic ischemia stage. J Mal Vasc 2000;25(1):17-26. 